OPEN ACCESS

Edited by:

Bingyun Li,

West Virginia University, United States

Reviewed by:

Anna Zemke,

University of Pittsburgh, United States Jessica Amber Jennings, University of Memphis, United States

*Correspondence:

Mercedes Gonzalez Moreno mercedes.gonzalez-moreno@charite.de

Specialty section:

This article was submitted to Antimicrobials, Resistance and

Chemotherapy,

a section of the journal

Frontiers in Microbiology

Received: 01 August 2019 Accepted: 18 October 2019

Published: 06 November 2019

Citation:

Wang L, Di Luca M, Tkhilaishvili T, Trampuz $A$ and Gonzalez Moreno M

(2019) Synergistic Activity

of Fosfomycin, Ciprofloxacin, and Gentamicin Against Escherichia coli and Pseudomonas aeruginosa Biofilms.

Front. Microbiol. 10:2522. doi: 10.3389/fmicb.2019.02522

\section{Synergistic Activity of Fosfomycin, Ciprofloxacin, and Gentamicin Against Escherichia coli and Pseudomonas aeruginosa Biofilms}

\author{
Lei Wang ${ }^{1,2}$, Mariagrazia Di Luca', Tamta Tkhilaishvili',2, Andrej Trampuz ${ }^{1,2}$ and \\ Mercedes Gonzalez Moreno ${ }^{1,2 *}$
}

${ }^{1}$ Center for Musculoskeletal Surgery, Charité - Universitätsmedizin Berlin, Corporate Member of Freie Universität Berlin, Humboldt-Universität zu Berlin and Berlin Institute of Health, Berlin, Germany, ${ }^{2}$ Berlin-Brandenburg Center for Regenerative Therapies, Charité - Universitätsmedizin Berlin, Berlin, Germany

Gram-negative (GN) rods cause about 10\% periprosthetic joint infection (PJI) and represent an increasing challenge due to emergence of antimicrobial resistance. Escherichia coli and Pseudomonas aeruginosa are among the most common cause of GN-PJI and ciprofloxacin is the first-line antibiotic. Due to emergence of fluoroquinolone resistance, we evaluated in vitro the activity of fosfomycin, ciprofloxacin, and gentamicin, alone and in combinations, against $E$. coli and $P$. aeruginosa biofilms. Conventional microbiological tests and isothermal microcalorimetry were applied to investigate the anti-biofilm activity of the selected antibiotics against standard laboratory strains as well as clinical strains isolated from patients with prosthetic joint associated infections. The biofilm susceptibility to each antibiotic varied widely among strains, while fosfomycin presented a poor antibiofilm activity against $P$. aeruginosa. Synergism of two-pair antibiotic combinations was observed against different clinical strains from both species. Highest synergism was found for the fosfomycin/gentamicin combination against the biofilm of E. coli strains (75\%), including a gentamicin-resistant but fosfomycin-susceptible strain, whereas the gentamicin/ ciprofloxacin combination presented synergism with higher frequency against the biofilm of $P$. aeruginosa strains (71.4\%). A hypothetical bacteriolysis effect of gentamicin could explain why combinations with this antibiotic seem to be particularly effective. Still, the underlying mechanism of the synergistic effect on biofilms is unknown. In conclusion, combinatorial antibiotic application has shown to be more effective against biofilms compared to monotherapy. Further in vivo and clinical studies are essential to define the potential treatment regimen based on our results.

Keywords: Escherichia coli, Pseudomonas aeruginosa, biofilm-associated infection, antibiotic activity, synergism, clinical isolates, antibiotic resistance, isothermal microcalorimetry

\section{INTRODUCTION}

Gram-negative (GN) rods cause about 10\% of periprosthetic joint infection (PJI) and represent an increasing treatment challenge due to the emergence of resistance worldwide (Shah et al., 2016; Thompson et al., 2018). Enterobacteriaceae are most frequently isolated in GN-PJI, followed by Pseudomonas aeruginosa (Fantoni et al., 2019). GN-PJI can occur after hematogenous 
seeding from a distant infectious focus (i.e. urinary or intestinal tract) or can be introduced during arthroplasty and manifest in the early postoperative period (Sendi et al., 2010). Over a period of a few years (2003-2012), the occurrence of PJIs due to multidrug-resistant GN bacteria has increased significantly, in the case of E. coli, from 2 to 4.3\%, and for P. aeruginosa, from 0.7 to $1.8 \%$ (Benito et al., 2016). Antimicrobial resistance in GN rods is increasing at both, community and hospital levels, and is often associated with treatment failure (Virginio et al., 2019). The worldwide rise of carbapenem-resistant GN bacilli is of major concern for the public health. While formally this problem was mostly related to Pseudomonas and Acinetobacter species, the rising trend in $E$. coli may lead to almost untreatable community-acquired infections (Tangden and Giske, 2015).

In spite of its vast impact on patients and the health-care system (Haddad et al., 2017), the management of GN-PJI is difficult due to the lack of a "gold standard" treatment strategy (Zmistowski et al., 2011; Goel et al., 2017). In fluoroquinolonesusceptible GN rods, ciprofloxacin is recommended for PJI (Sousa and Abreu, 2018). However, the growing quinoloneresistance in GN bacteria makes the treatment of GN-PJI more challenging complicating the clinical outcome (Rodriguez-Pardo et al., 2014; Benito et al., 2016). Due to unmet medical needs of currently available antibiotics, combination therapy has been investigated as an alternative strategy for GN-PJI treatment (Taha et al., 2018). Especially, revival of older antibiotics such as fosfomycin gained attention for treatment of multi-drug resistant GN rods (Walsh et al., 2016).

Beside antimicrobial resistance, treatment of PJI is challenged by the microbial persistence on the surface of implants forming biofilms (Taha et al., 2018). In biofilms, microbes exhibit "phenotypical resistance" to standard antibiotics (Donlan and Costerton, 2002). Therefore, it is essential to look at possible anti-biofilm activities of single or combined antibiotics. Several pre-clinical investigations of fosfomycin combination therapy have shown synergistic activity against biofilms of GN bacteria (Falagas et al., 2016), particularly in combination with fluoroquinolones or aminoglycosides (Michalopoulos et al., 2011; Corvec et al., 2013). Nevertheless, no systematic studies investigated these combinations against $P$. aeruginosa and Escherichia coli biofilms in the same experimental settings. Thus, we focused on combinations involving fosfomycin, ciprofloxacin, and gentamicin as representatives of the abovementioned antibiotic classes. These three antibiotics present a bactericidal effect against bacteria showing different mechanisms of action. Fosfomycin has a unique mode of action inhibiting irreversibly an early stage of bacterial cell wall biosynthesis (Dijkmans et al., 2017), whereas ciprofloxacin inhibits bacterial DNA replication (Thai and Zito, 2019) and gentamicin inhibits the bacterial protein synthesis (Kumar et al., 2008).

Accurate experimental data from the investigation of combinatorial therapy with paired antibiotics might bring new evidences on their potential in the treatment of GN-PJI. Hence, we evaluated the in vitro activity of single and combinations of fosfomycin, ciprofloxacin, and gentamicin against planktonic and biofilms of $P$. aeruginosa and $E$. coli strains, including resistant clinical isolates obtained from patients with prosthetic joint associated infections, by using conventional microbiological tests and isothermal microcalorimetry.

\section{MATERIALS AND METHODS}

\section{Bacterial Strains}

E. coli (ATCC 25922) and P. aeruginosa (ATCC 27853) laboratory standard strains were used in this study. Moreover, eight $E$. coli and seven $P$. aeruginosa clinical isolates obtained from consecutive patients diagnosed with PJI between 2015 and 2017 were used for this study. For the diagnosis of PJI, the PRO-IMPLANT diagnostic criteria were used ( $\mathrm{Li}$ et al., 2018; Izakovicova et al., 2019). The clinical isolates were used from the biobank collection, which is part of the prospective institutional PJI cohort. The study was approved by the institutional ethical committee (EA1/040/14) and was conducted in accordance with the most recent iteration of the Declaration of Helsinki. According to the ethical approval, participants' informed consent was waived and all data were pseudonymized. Bacteria were stored at $-80^{\circ} \mathrm{C}$ using a cryovial bead preservation system (Microbank; Pro-Lab Diagnostics, Canada).

\section{Antimicrobial Agents}

Fosfomycin (5 g; InfectoPharm, Heppenheim, Germany) was provided as purified powder by the manufacturer. Ciprofloxacin injectable solution $(2 \mathrm{mg} / \mathrm{ml}$; Fresenius $\mathrm{Kabi} \mathrm{GmbH}$, Bad Homburg, Germany) and gentamicin injectable solution (40 mg/ $\mathrm{ml}$; Ratiopharm GmbH, Ulm, Germany) were purchased from the respective manufacturers. Stock solutions of appropriate concentrations were prepared in sterile $0.9 \%$ saline.

\section{Etest}

Etest (bioMerieux, Marcy-l'Étoile, France) was performed in Mueller-Hinton agar (MHA) (Becton, Dickinson and Company, Germany) following the manufacturer's instructions. The minimum inhibitory concentration (MIC) was determined as the concentration at which the inhibition ellipse intersected the scale of the strip after incubation at $37^{\circ} \mathrm{C}$ for $24 \mathrm{~h}$. To evaluate the susceptibility, the antimicrobial susceptibility breakpoints from the CLSI (CLSI, 2015) were used. All experiments were performed in triplicates.

\section{Broth Macrodilution Assays}

The MIC and the minimum bactericidal concentration (MBC) phase were determined for fosfomycin, ciprofloxacin, and gentamicin by the broth macrodilution assay (BMD) in cationadjusted Mueller-Hinton broth (CAMHB) (BD, Le Pont de Claix, France), according to the Clinical and Laboratory Standards Institute (CLSI) guidelines (CLSI, 1999). An inoculum of approximately $5 \times 10^{5} \mathrm{CFU} / \mathrm{ml}$ were used. Two-fold serial dilutions of each antibiotic were prepared in $1 \mathrm{ml}$ medium in plastic tubes and incubated for $24 \mathrm{~h}$ at $37^{\circ} \mathrm{C}$. The MIC 
was defined as the lowest concentration of antibiotic that completely inhibited visible growth.

After the incubation, all tubes without visible growth were vigorously vortexed, aliquots of $100 \mu \mathrm{l}$ were plated on Tryptic Soy Agar (TSA) (Oxoid, Basingstoke, UK) plates, and the numbers of bacteria were determined. The MBC was defined as the lowest antimicrobial concentration that killed $\geq 99.9 \%$ of the initial bacterial inoculum after $24 \mathrm{~h}$. The medium was supplemented with $25 \mathrm{mg} / \mathrm{L}$ glucose-6-phosphate for testing of fosfomycin. Glucose-6-phosphate induces the transport system via which fosfomycin is actively absorbed into the bacteria (Dijkmans et al., 2017). All experiments were performed in triplicates.

\section{Assessment of Antimicrobial Activity by Isothermal Microcalorimetry and Sonication/Colony-Counting}

The antimicrobial activity of fosfomycin, ciprofloxacin, and gentamicin against either $E$. coli or P. aeruginosa ATCC strains was determined by isothermal microcalorimetry (IMC) as described previously (Butini et al., 2018). Briefly, planktonic bacteria $\left(5 \times 10^{5} \mathrm{CFU} / \mathrm{ml}\right)$ were treated with serial dilutions of antibiotics in CAMHB, and production of heat was measured for $24 \mathrm{~h}$. The minimum heat inhibitory concentration (MHIC) was defined as the lowest concentration of antibiotic able to suppress the metabolic heat production of planktonic bacteria.

E. coli and $P$. aeruginosa biofilm formation was assessed by incubating porous glass beads ( $\mathrm{ROBU}^{\circledR}$, Hattert, Germany) in inoculated CAMHB with 2-3 colonies of the corresponding bacteria at $37^{\circ} \mathrm{C}$. The ratio between beads and diluted bacterial suspension was 1 bead: $1 \mathrm{ml}$, with a maximum of 10 beads per $50 \mathrm{ml}$ Falcon tube. After $24 \mathrm{~h}$ incubation, beads were washed three times with sterile $0.9 \%$ saline to remove planktonic bacteria and exposed to serial dilutions of antibiotic in $1 \mathrm{ml}$ of CAMHB and incubated for a further $24 \mathrm{~h}$ at $37^{\circ} \mathrm{C}$. The media were supplemented with $25 \mathrm{mg} / \mathrm{L}$ glucose-6-phosphate for fosfomycin testing. After exposure to antibiotics, beads were washed three times with $0.9 \%$ saline, placed in glass ampoules containing $3 \mathrm{ml}$ of CAMHB and introduced into the calorimeter (thermal activity monitor, model 3102 TAM III; TA Instruments, New Castle, USA). Sterile beads were used as negative control. Production of heat was recorded for $48 \mathrm{~h}$ to detect bacterial activity. The minimum biofilm bactericidal concentration (MBBC) was defined as the lowest concentration of antibiotic that strongly reduced biofilm cells viability and led to the absence of heat flow production after $48 \mathrm{~h}$ of incubation at $37^{\circ} \mathrm{C}$.

Moreover, the biofilm-eradicating activity of these three antibiotics on clinical isolates from both species was evaluated by sonication and colony-counting as in a previous study (Gonzalez Moreno et al., 2019). The minimum biofilm eradicating concentration (MBEC) was defined as the lowest concentration of antibiotic required to kill all sessile cells resulting in the appearance of no colony after plating sonication fluid (detection limit: $<20 \mathrm{CFU} / \mathrm{ml})$.

The synergistic effect of antibiotic combinations was evaluated against both ATCC species following the IMC assay as described above and through CFU counting of the sonicated beads. The synergistic activity was evaluated by calculation of the fractional biofilm eradication concentration index (FBECI) as described in a previous study (Dall et al., 2018), where a FBECI of $\leq 0.5$ indicates a synergistic effect. The FBECI was calculated following the equation: FBECI $=$ FBECI $\mathrm{A}+\mathrm{FBECI} \mathrm{B}=\mathrm{MBEC}_{\mathrm{A}}$ combination $/$ $\mathrm{MBEC}_{\mathrm{A}}$ alone $+\mathrm{MBEC}_{\mathrm{B}}$ combination/MBEC $\mathrm{B}_{\mathrm{B}}$ alone, where $\mathrm{MBEC}_{\mathrm{A}}$ combination and $\mathrm{MBEC}_{\mathrm{B}}$ combination are the MBEC of compound $\mathrm{A}$ in the presence of $\mathrm{B}$ and compound $\mathrm{B}$ in the presence of $A$, respectively; $M B E C_{A}$ alone and $M B E C_{B}$ alone are the FBECI of compound $\mathrm{A}$ and compound $\mathrm{B}$, respectively.

Data from IMC were analyzed by the manufacturer's software (TAM Assistant; TA Instruments) and Prism 7.0 (GraphPad Software, La Jolla, CA). All experiments were performed in triplicates.

\section{RESULTS}

\section{Activity of Antibiotics Against E. coli and $P$. aeruginosa ATCC Strains}

The antimicrobial activity of fosfomycin, ciprofloxacin, and gentamicin against planktonic and sessile E. coli and P. aeruginosa ATCC strains was assessed using BMC, Etest, IMC (Figures 1, 2) and by plating of sonication fluid. Table 1 summarizes the susceptibilities of both species.

The observed MIC values evaluated by Etest and BMD were comparable to those obtained by IMC. Both ATCC strains were susceptible to all three tested antibiotics. Ciprofloxacin was the most active antibiotic against planktonic bacteria from both strains, followed by gentamicin. Fosfomycin showed a remarkable lower bactericidal activity against $P$. aeruginosa with MIC and MBC values 16 and 64 times higher respectively compared to E. coli.

Gentamicin was the most active antibiotic against the biofilm of both strains presenting a MBBC of $16 \mu \mathrm{g} / \mathrm{ml}$ (Figures 2E,F), whereas ciprofloxacin showed a notable higher anti-biofilm activity against E. coli $(\mathrm{MBBC}=16 \mu \mathrm{g} / \mathrm{ml})$ compared to $P$. aeruginosa $(\mathrm{MBBC}=512 \mu \mathrm{g} / \mathrm{ml}$ ) (Figures 2C,D). Fosfomycin exhibited a poor anti-biofilm activity against both tested ATCC strains (Figures 2A,B).

Results showed that the concentrations of antibiotics necessary to completely eradicate the biofilm (MBEC) of both ATCC strains correlated with the bactericidal concentrations observed by calorimetry (MBBC) for all the tested antibiotics.

\section{Anti-biofilm Activity of Combined Antibiotics Against E. coli and $P$. aeruginosa ATCC Strains}

The synergistic effect of two-pair antibiotics against biofilm of both ATCC strains was investigated by IMC combining fosfomycin/ ciprofloxacin, fosfomycin/gentamicin, and gentamicin/ciprofloxacin. Results are summarized in Table 2. Calorimetric curves are depicted in Figure 3. 

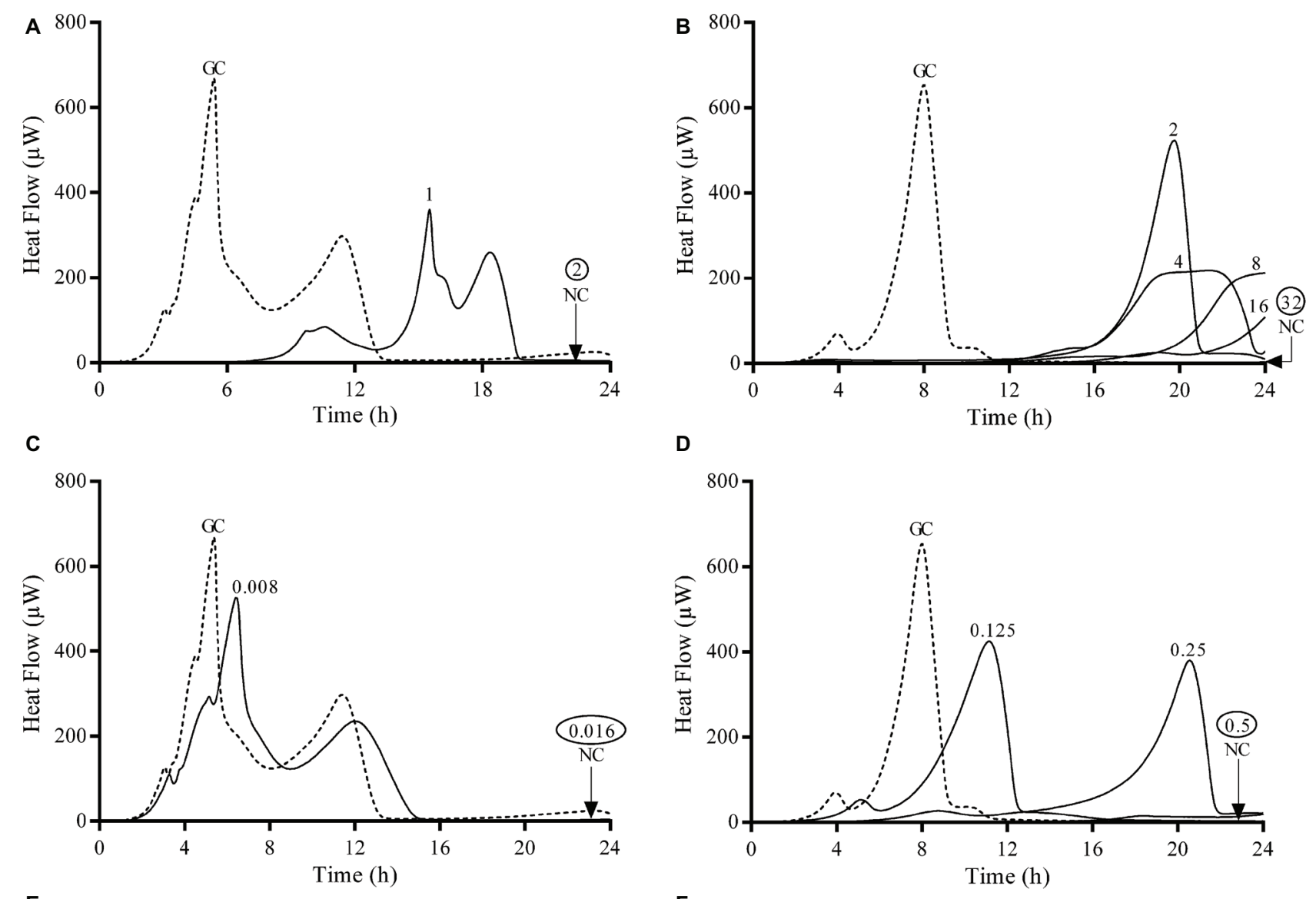

D
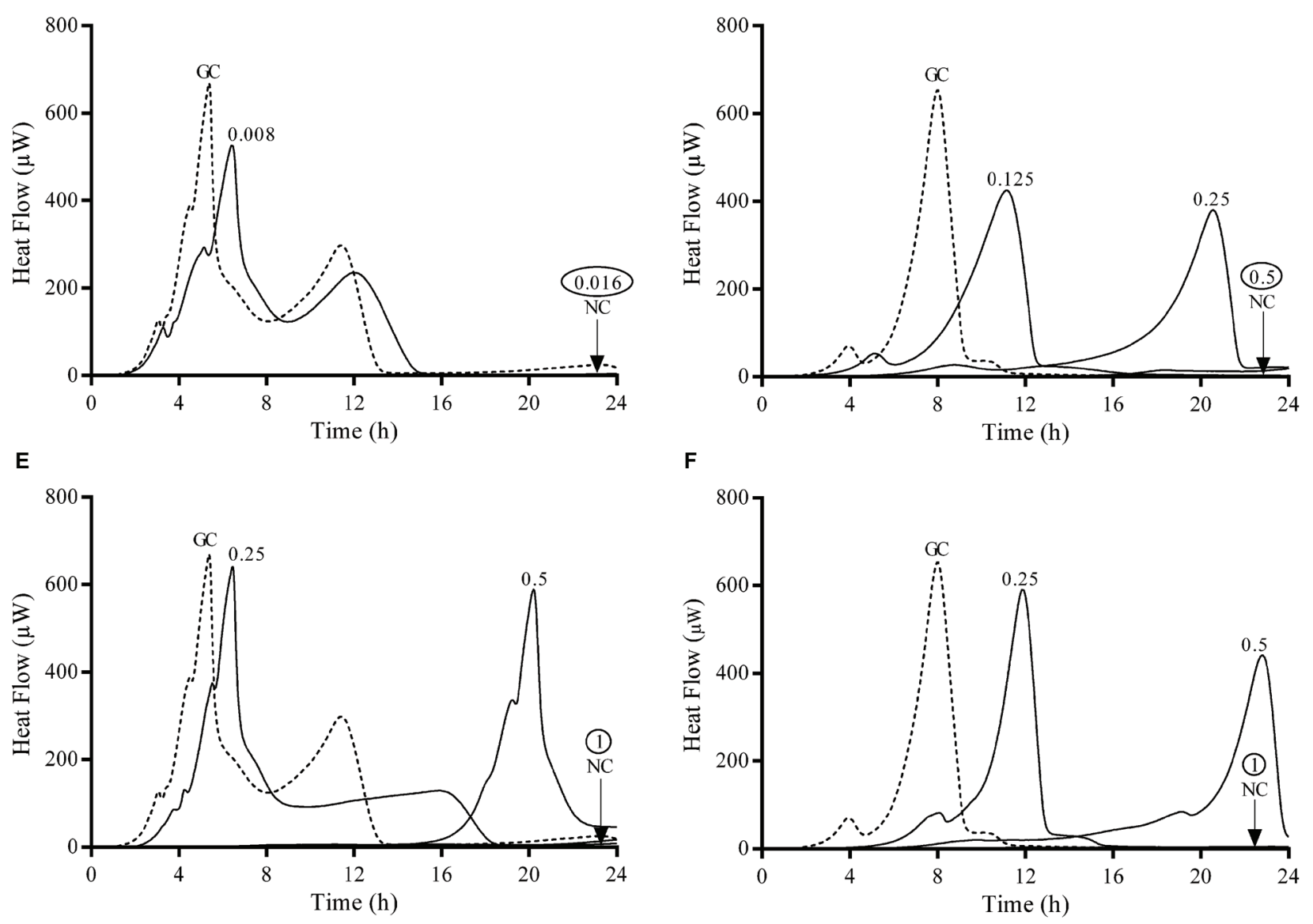

FIGURE 1 | Microcalorimetry analysis of planktonic bacteria exposed to serial antibiotic concentrations for $24 \mathrm{~h}$. Numbers represent concentrations (in $\mu \mathrm{g} / \mathrm{ml}$ ) of fosfomycin (A,B), ciprofloxacin (C,D), and gentamicin (E,F) against E. coli ATCC 25922 (left column) and P. aeruginosa ATCC 27853 (right column). Circled values represent the MHIC. GC, growth control; NC, negative control. Data of a representative experiment are reported.

The strongest synergistic effect was observed when gentamicin was combined with fosfomycin $(\mathrm{FBEC}=0.06)$ against $E$. coli biofilm followed by the combination of gentamicin with ciprofloxacin, whereas these two antibiotic combinations showed similar synergistic effect against $P$. aeruginosa biofilm. Fosfomycin/ ciprofloxacin combination did not show synergism against the biofilm of both strains.

\section{Antibiotic Susceptibility of $E$. coli and $\boldsymbol{P}$ aeruginosa Clinical Strains}

The MIC of E. coli and P. aeruginosa clinical strains to fosfomycin, ciprofloxacin, and gentamicin was determined by Etest. The results are summarized in Tables 3 and 4 .

E. coli and $P$. aeruginosa strains were considered susceptible to fosfomycin when $\mathrm{MIC} \leq 64 \mu \mathrm{g} / \mathrm{ml}$, to ciprofloxacin when 

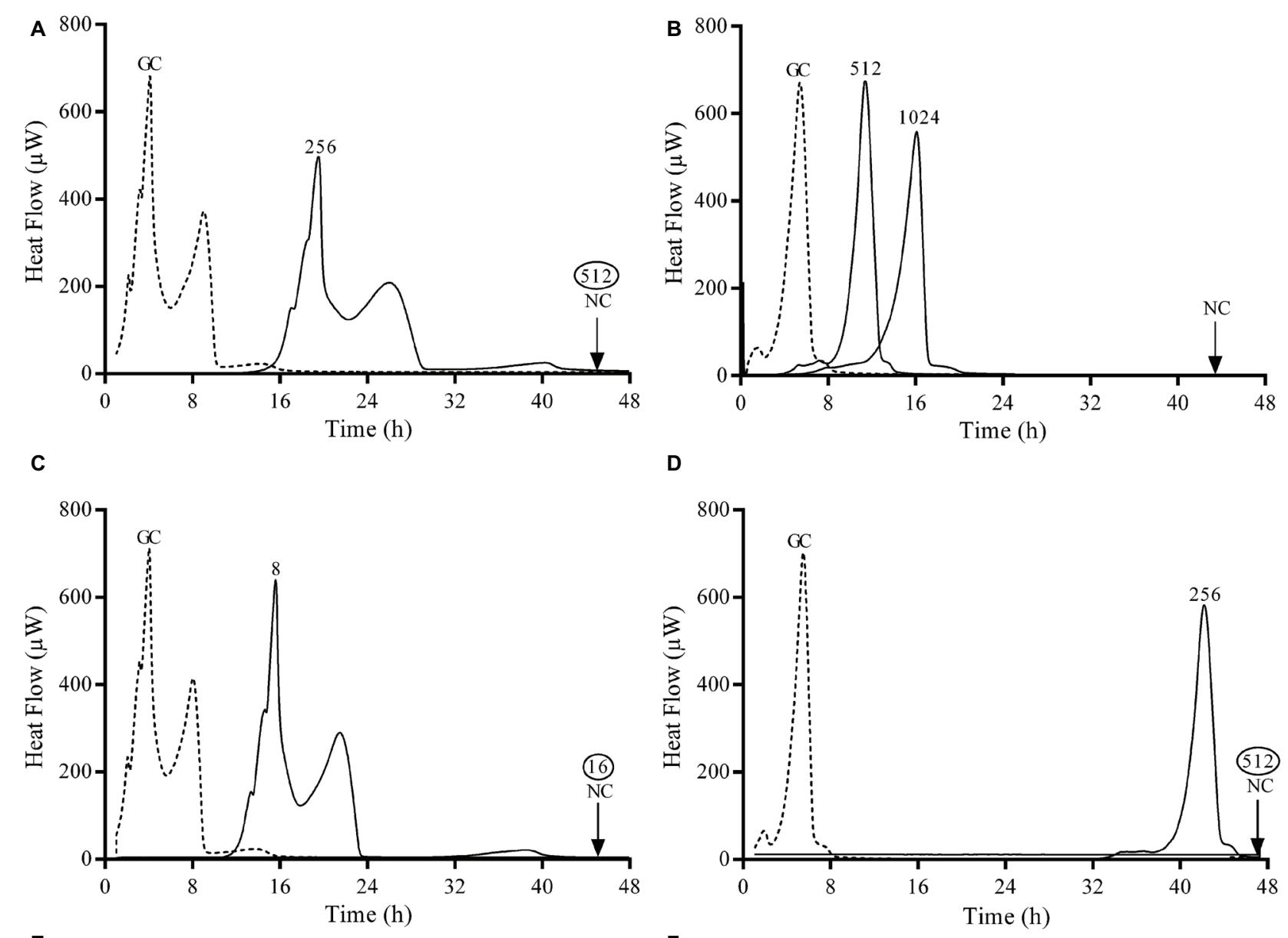

D
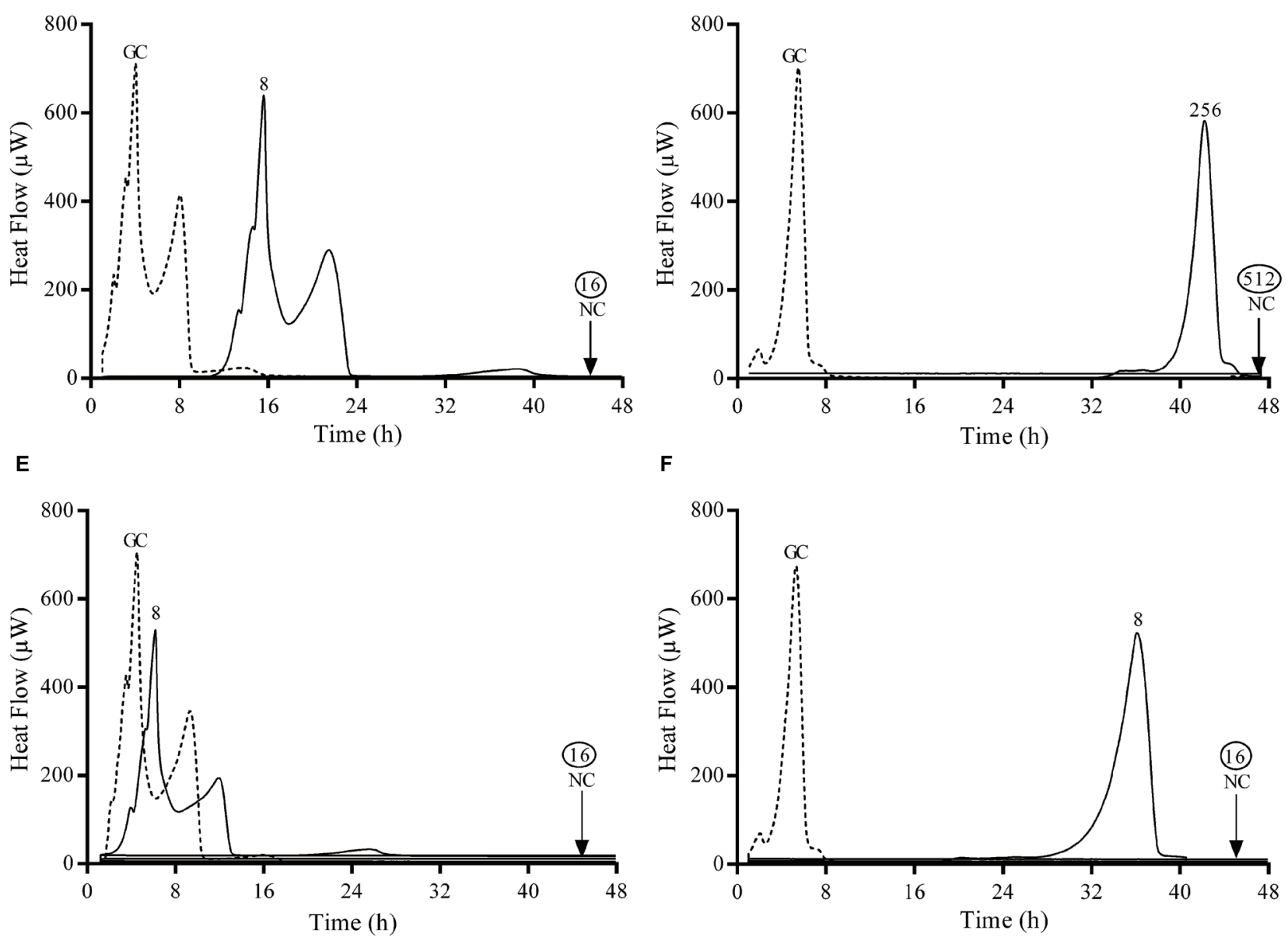

FIGURE 2 | Microcalorimetry analysis of biofilm bacteria after exposure to serial antibiotic concentrations for $24 \mathrm{~h}$. Numbers represent concentrations (in $\mu \mathrm{g} / \mathrm{ml}$ ) of fosfomycin (A,B), ciprofloxacin (C,D), and gentamicin (E,F) against E. coli ATCC 25922 (left column) and P. aeruginosa ATCC 27853 (right column). Circled values represent the MBBC. GC, growth control; NC, negative control. Data of a representative experiment are reported.

$\mathrm{MIC} \leq 1 \mu \mathrm{g} / \mathrm{ml}$ and to gentamicin when $\mathrm{MIC} \leq 4 \mu \mathrm{g} / \mathrm{ml}$ according to CLSI (CLSI, 2015).

Most strains were susceptible to the tested antibiotics, except Ec6 (resistant to ciprofloxacin and gentamicin), Ec7 (resistant to fosfomycin and ciprofloxacin), Ec8 (resistant to fosfomycin and gentamicin), $\mathrm{Pa} 6$ (resistant to gentamicin), and $\mathrm{Pa} 7$ (resistant to ciprofloxacin).

Ciprofloxacin exhibited the lowest MIC in sensitive strains for both bacterial species (MIC range $0.008-0.25 \mu \mathrm{g} / \mathrm{ml}$ ), whereas fosfomycin showed higher activity on susceptible strains of 
TABLE 1 | Antimicrobial susceptibility of planktonic and adherent E. coli and $P$. aeruginosa determined by conventional broth macrodilution (BMD), Etest, isothermal microcalorimetry (IMC), and sonication/colony-counting.

\begin{tabular}{|c|c|c|c|c|c|c|}
\hline \multicolumn{7}{|c|}{ E. coli ATCC 25922} \\
\hline \multirow{2}{*}{ Antibiotic } & \multirow{2}{*}{$\begin{array}{l}\text { Etest } \\
\text { MIC }\end{array}$} & \multicolumn{2}{|c|}{ BMD } & \multicolumn{2}{|c|}{ IMC } & \multirow{2}{*}{$\begin{array}{c}\text { Sonication } \\
\text { MBEC }\end{array}$} \\
\hline & & MIC & MBC & MHIC & MBBC & \\
\hline Fosfomycin & 1 & 2 & 4 & 2 & 512 & 512 \\
\hline Ciprofloxacin & 0.012 & 0.008 & 0.016 & 0.016 & 16 & 16 \\
\hline Gentamicin & 0.5 & 0.5 & 4 & 1 & 16 & 16 \\
\hline \multicolumn{7}{|c|}{ P. aeruginosa ATCC 27853} \\
\hline \multirow{2}{*}{ Antibiotic } & Etest & \multicolumn{2}{|c|}{ BMD } & \multicolumn{2}{|c|}{ IMC } & Sonication \\
\hline & MIC & MIC & MBC & MHIC & MBBC & MBEC \\
\hline Fosfomycin & 16 & 32 & 256 & 32 & $>1,024$ & $>1,024$ \\
\hline Ciprofloxacin & 0.25 & 0.25 & 1 & 0.5 & 512 & 512 \\
\hline Gentamicin & 1 & 0.5 & 4 & 1 & 16 & 16 \\
\hline
\end{tabular}

Concentration values are expressed in $\mu \mathrm{g} / \mathrm{ml}$.

TABLE 2 | MBBC and FBEC values for fosfomycin (FOS), ciprofloxacin (CIP), and gentamicin (GEN) in combination against E. coli and P. aeruginosa.

\begin{tabular}{|c|c|c|c|c|}
\hline \multirow[t]{2}{*}{ Antibiotic } & \multicolumn{2}{|c|}{ E. coli (ATCC 25922) } & \multicolumn{2}{|c|}{ P. aeruginosa (ATCC 27853) } \\
\hline & MBBC $(\mu \mathrm{g} / \mathrm{ml})$ & FBEC (interpretation) & MBBC ( $\mu \mathrm{g} / \mathrm{ml})$ & FBEC (interpretation) \\
\hline $\mathrm{FOS}+\mathrm{CIP}$ & $128+8$ & 0.75 (NS) & $256+256$ & $0.75^{\star}(\mathrm{NS})$ \\
\hline $\mathrm{FOS}+\mathrm{GEN}$ & $2+1$ & $0.06(S)$ & $256+2$ & $0.38^{*}(\mathrm{~S})$ \\
\hline GEN + CIP & $1+2$ & $0.19(\mathrm{~S})$ & $4+8$ & $0.26(\mathrm{~S})$ \\
\hline
\end{tabular}

MBBC, minimal biofilm bactericidal concentration; FBEC, fractional biofilm eradication concentration; S, synergism, NS, no synergism; ${ }^{\star M B B C}$ Fos of P. aeruginosa was considered equal to $1,024 \mu \mathrm{g} / \mathrm{ml}$ for FBEC calculations.

E. coli (MIC range $0.064-1 \mu \mathrm{g} / \mathrm{ml})$ than $P$. aeruginosa $(8-48 \mu \mathrm{g} /$ $\mathrm{ml})$. Gentamicin-susceptible strains from both species presented similar susceptible profile with a MIC range of $0.5-3 \mu \mathrm{g} / \mathrm{ml}$.

\section{Synergistic Effect of Antibiotic Combinations Against E. coli and $P$. aeruginosa Clinical Strains}

The same two-pair antibiotic combinations tested against the ATCC strains were used to evaluate their ability to eradicate biofilms from clinical strains by sonication/colony counting. Tables 5 and 6 summarize the results of the MBEC for single and combined antibiotics against $E$. coli and P. aeruginosa clinical strains.

The biofilm susceptibility to each antibiotic varied widely among clinical isolates. Among the eight tested E. coli isolates, synergism based on fosfomycin/ciprofloxacin combinations was observed in two isolates (25\%), while the combinations fosfomycin/gentamicin and gentamicin/ ciprofloxacin resulted synergistic in six (75\%) and three isolates $(37.5 \%)$, respectively.

Moreover, the fosfomycin/gentamicin combination showed a synergistic effect against Ec6, a gentamicin-resistant but fosfomycin-susceptible $E$. coli strain, whereas the synergistic effect was not observed when the fosfomycin/gentamicin combination was tested against Ec7, a fosfomycin-resistant but gentamicin-susceptible $E$. coli strain.
On the other hand, the synergism of gentamicin/ciprofloxacin was observed in five $P$. aeruginosa isolates (71.4\%), while four isolates $(57.1 \%)$ were susceptible to the combination of either fosfomycin/ciprofloxacin or fosfomycin/gentamicin.

\section{DISCUSSION}

Fluoroquinolones are the first choice as anti-biofilm antibiotics for the treatment of GN-PJI (Boyle et al., 2019). However, emergence and spread of resistance to fluoroquinolones and aminoglycosides has decreased the existing treatment options for GN infections (Tucaliuc et al., 2015). Combination therapy with fosfomycin has been recommended, particularly against fluoroquinolone resistant organisms (Boyle et al., 2019). Nonetheless, there is a lack of systematic studies investigating antibiotic combinations under the same experimental settings on GN biofilms. In this study, we have generated original data showing synergistic activity of two-pair antibiotic combinations against either $E$. coli or $P$. aeruginosa biofilms in vitro.

Conventional (Etest, BMD and colony counting) and nonconventional (IMC) laboratory tests were applied to evaluate the susceptibility to antibiotics of either planktonic or biofilm bacteria. As seen also in previous studies (Gonzalez Moreno et al., 2017, 2019), the MHIC values obtained by IMC showed consistency to the MIC values obtained by BMD and Etest, proving the reliability of IMC for antimicrobial testing on large 

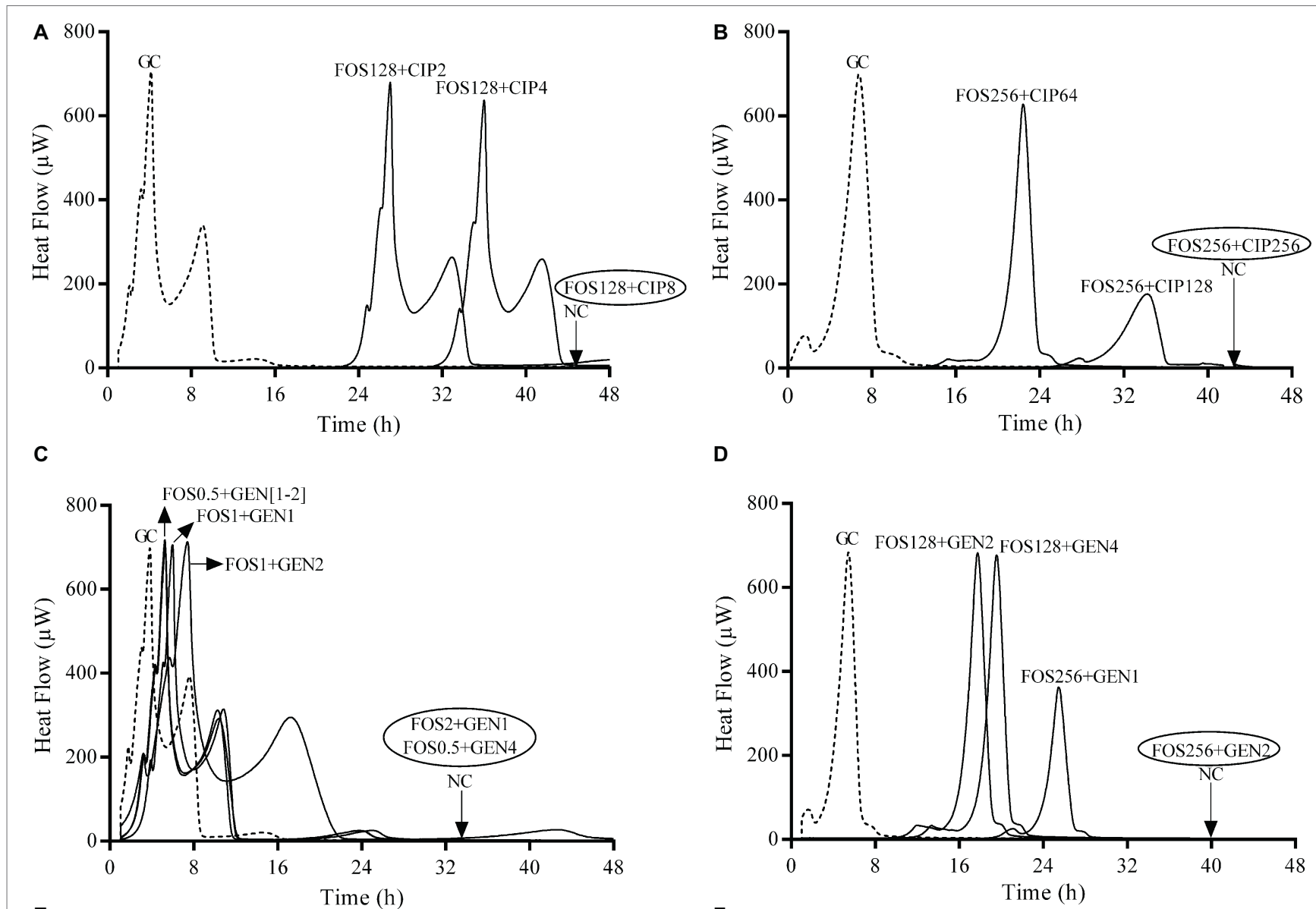

D

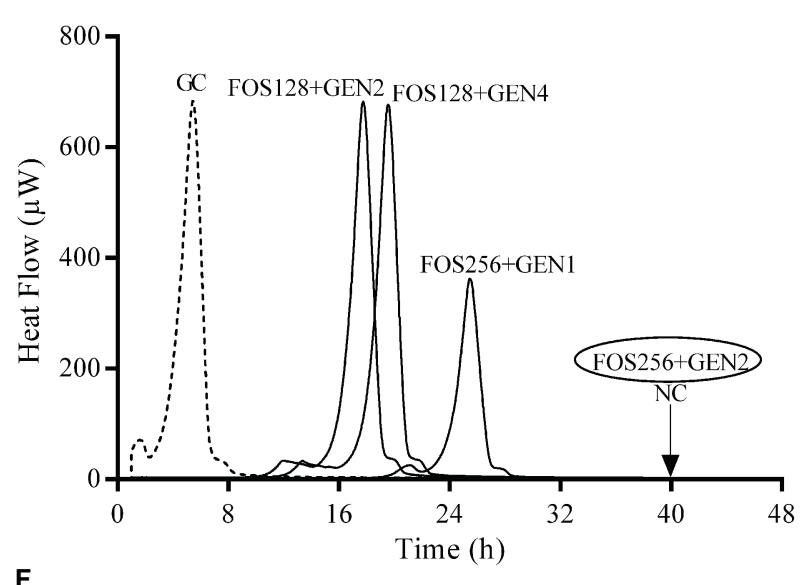

E
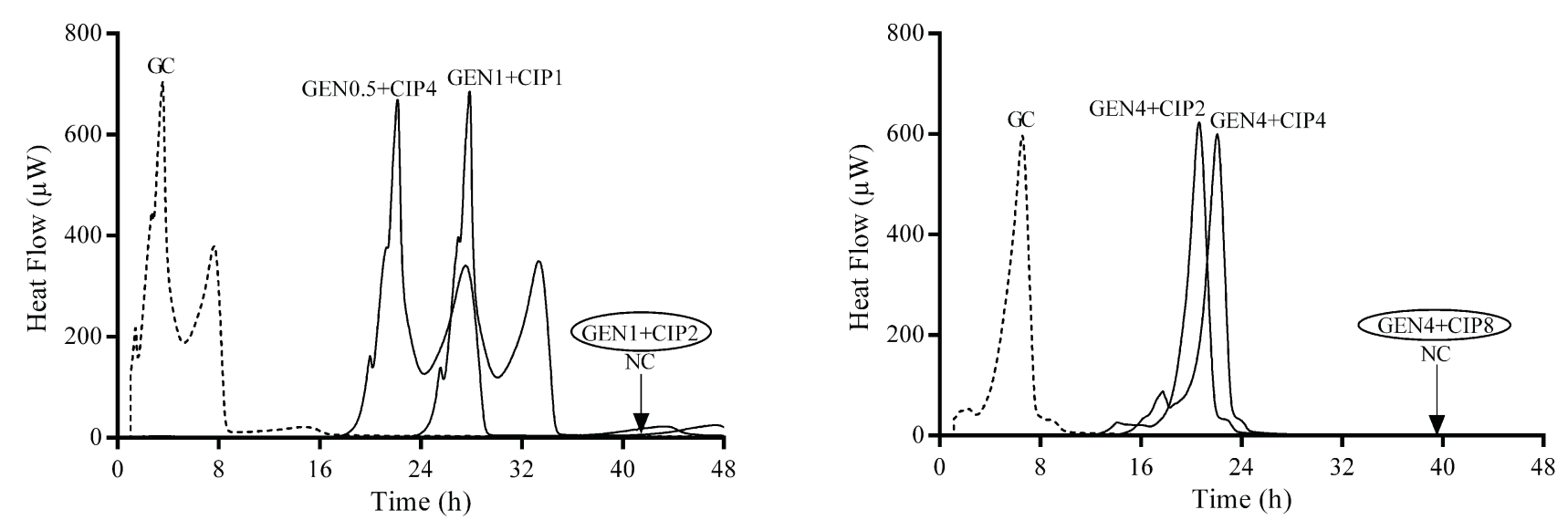

FIGURE 3 | Evaluation of synergistic activity of paired antibiotics by IMC against E. coli ATCC 25922 (A,C,E) and P. aeruginosa ATCC 27853 (B,D,F) biofilms. Numbers represent concentrations (in $\mu \mathrm{g} / \mathrm{ml}$ ). Circled values represent the MBBC. GC, growth control; NC, negative control; FOS, fosfomycin; GEN, gentamicin; $\mathrm{CIP}$, ciprofloxacin. Data of a representative experiment are reported.

scale. Moreover, the concentrations of antibiotics needed to eradicate biofilms were analogous to those showing biofilm bactericidal activity by IMC.

As already reported for many microorganisms (Stewart, 2015), in our study, the eradication of GN biofilms required considerably higher concentrations of all three tested antibiotics (4 to 2,723-fold higher) compared with the killing of their planktonic bacteria. Fosfomycin had no anti-biofilm activity against $P$. aeruginosa strains despite the use of high concentrations of antibiotic (up to $1,024 \mu \mathrm{g} / \mathrm{ml}$ ) (Table 6). These results suggest that outcomes obtained on planktonic cells cannot be transferred to biofilms, underling the importance of developing standardize methods to evaluate antimicrobial activity on biofilms (Macia et al., 2014). In our work, we employed IMC in 
combination with sonication as reliable methods for the in vitro analysis of bacterial biofilms (Butini et al., 2018).

In biofilm infections, where antimicrobial monotherapies are not effective or not applicable due to the rapid development of antibiotic resistance (Wu et al., 2015; Greimel et al., 2017; $\mathrm{Xu}$ et al., 2018), the use of antibiotic combinatorial therapies has been shown to be particularly relevant due to the potential synergy between drugs (Wu et al., 2015). Therefore, we also

TABLE 3 | MIC of E. coli by Etest.

\begin{tabular}{lcccccccc}
\hline Antimicrobial & Ec1 & Ec2 & Ec3 & Ec4 & Ec5 & Ec6 & Ec7 & Ec8 \\
\hline Fosfomycin & 0.19 & 0.094 & 0.125 & 0.064 & 0.75 & 0.25 & $128(\mathrm{R})$ & $64(\mathrm{R})$ \\
Ciprofloxacin & 0.016 & 0.016 & 0.008 & 0.008 & 0.008 & $8(\mathrm{R})$ & $8(\mathrm{R})$ & 0.008 \\
Gentamicin & 1 & 0.5 & 1 & 2 & 1 & $96(\mathrm{R})$ & 1 & $96(\mathrm{R})$ \\
\hline
\end{tabular}

MIC values are expressed in $\mu \mathrm{g} / \mathrm{ml}$; $R$, resistant (according to CLSI).

TABLE 4 | MIC of $P$. aeruginosa by Etest.

\begin{tabular}{lccccccc}
\hline Antimicrobial & Pa1 & Pa2 & Pa3 & Pa4 & Pa5 & Pa6 & Pa7 \\
\hline Fosfomycin & 32 & 48 & 8 & 16 & 32 & 24 & 48 \\
Ciprofloxacin & 0.19 & 0.064 & 0.125 & 0.125 & 0.094 & 0.19 & $12(\mathrm{R})$ \\
Gentamicin & 1.5 & 1.5 & 2 & 3 & 2 & $128(\mathrm{R})$ & 1.5 \\
\hline
\end{tabular}

MIC values are expressed in $\mu \mathrm{g} / \mathrm{ml}$; $R$, resistant (according to CLSI). investigated the in vitro synergistic activity of paired antibiotics against $E$. coli and P. aeruginosa ATCC strains and clinical isolates.

A study limitation is the lack of a full chequerboard analysis for antibiotic combinations, which could bring more insights on the synergistic/antagonistic activity. We evaluated only concentrations of antibiotics that could reveal a synergistic effect based on the MBEC values of the single antibiotics to be combined. For MBEC $>1,024 \mu \mathrm{g} / \mathrm{ml}$, a fixed value of $1,024 \mu \mathrm{g} / \mathrm{ml}$ was considered for the calculation of the FBEC index, thus some combinations which were interpreted as not synergistic could turn out to have a synergistic effect considering higher MBEC values. However, with this approach, the observed positive synergistic effects of antibiotic combinations are certain and usually presenting considerably lower MBEC values compared to the MBEC values of single antibiotics, which are difficult to reach in clinical practice.

The three tested antibiotic combinations showed synergistic activity, to varying degrees, against different clinical strains from both species partially differing from the results observed with the ATCC strains. The considerable reduction of MBEC values with antibiotic combinations ranging from 2 -fold to 16 -fold in case of $P$. aeruginosa strains or 2 -fold to 128 -fold in case of $E$. coli strains compared to single drug was predominantly in the range, which is achievable by intravenous or oral antibiotic administration (Dijkmans et al., 2017; Thabit et al., 2019).

Even though several studies have reported that fosfomycin showed an estimable synergistic effect, among others, with

TABLE 5 | MBEC for fosfomycin (FOS), ciprofloxacin (CIP), gentamicin (GEN), and their combinations against E. coli clinical strains.

\begin{tabular}{|c|c|c|c|c|c|c|}
\hline Strain & FOS & CIP & GEN & FOS + CIP & FOS + GEN & GEN + CIP \\
\hline Ec2 & 4 & 64 & 8 & $0.5+2(0.16, \mathrm{~S})$ & $0.5+1(0.25, \mathrm{~S})$ & $1+2(0.16, S)$ \\
\hline Ec3 & 16 & 0.032 & 8 & $4+0.016(0.75, N S)$ & $2+1(0.25, S)$ & $2+0.016(0.75, N S)$ \\
\hline Ec4 & 8 & 0.032 & 8 & $2+0.016(0.75, N S)$ & $2+0.5(0.31, S)$ & $2+0.016(0.75, \mathrm{NS})$ \\
\hline Ec7 & $>1,024$ & $>1,024$ & 4 & $>256^{\star}+256^{\star}(>0.5, \mathrm{NS})$ & $>256^{\star}+1(>0.5, N S)$ & $>1+256^{\star}(>0.5, N S)$ \\
\hline Ec8 & $>1,024$ & 8 & $>1,024$ & $>256^{\star}+2(>0.5, \mathrm{NS})$ & $>256^{*}+256^{\star}(>0.5, \mathrm{NS})$ & $>256^{\star}+2(>0.5, N S)$ \\
\hline
\end{tabular}

MBEC, minimal biofilm eradication concentration (values are expressed in $\mu \mathrm{g} / \mathrm{mll}$. FBEC, fractional biofilm eradication concentration; $S$, synergism; NS, no synergism; *MBBC was considered equal to $1,024 \mathrm{\mu g} / \mathrm{ml}$ for FBEC calculations.

TABLE 6 | MBEC for fosfomycin (FOS), ciprofloxacin (CIP), gentamicin (GEN), and their combinations against $P$. aeruginosa clinical strains.

\begin{tabular}{|c|c|c|c|c|c|c|}
\hline Strain & FOS & CIP & GEN & FOS + CIP & FOS + GEN & GEN + CIP \\
\hline $\mathrm{Pa} 2$ & $>1,024$ & 32 & 32 & $256^{\star}+16(0.75, N S)$ & $64^{*}+4(0.19, S)$ & $4+2(0.19,5)$ \\
\hline $\mathrm{Pa} 3$ & $>1,024$ & 16 & 16 & $128^{\star}+2(0.25, S)$ & $128^{\star}+1(0.19, S)$ & $1+1(0.13, S)$ \\
\hline $\mathrm{Pa} 4$ & $>1,024$ & 8 & 16 & $32^{*}+2(0.28, \mathrm{~S})$ & $64^{\star}+1(0.13, S)$ & $4+1(0.38, S)$ \\
\hline $\mathrm{Pa} 7$ & $>1,024$ & $>1,024$ & 16 & $>256^{\star}+256^{\star}(>0.5, N S)$ & $>256^{\star}+4(>0.5, N S)$ & $>4+256^{*}(>0.5, \mathrm{NS})$ \\
\hline
\end{tabular}

MBEC, minimal biofilm eradication concentration (values are expressed in $\mu \mathrm{g} / \mathrm{ml}$ ). FBEC, fractional biofilm eradication concentration; S, synergism; NS, no synergism; ${ }^{*} M B B C$ was considered equal to $1,024 \mu \mathrm{g} / \mathrm{ml}$ for FBEC calculations. 
gentamicin and ciprofloxacin against $P$. aeruginosa planktonic cells (Kastoris et al., 2010), there is limited evidence for these combinations against biofilms. The fosfomycin/ciprofloxacin combination has being shown to be effective against $P$. aeruginosa biofilms in other experimental set-ups (Xiong et al., 1995; Mikuniya et al., 2005), whereas we could not find studies investigating the fosfomycin/ gentamicin combination. Nonetheless, previous studies have shown synergistic effect when fosfomycin was combined with other aminoglycosides (Cai et al., 2009; Anderson et al., 2013) as well as other fluoroquinolones (Kumon et al., 1995; Monden et al., 2002; Mikuniya et al., 2005, 2007) against P. aeruginosa biofilms.

Some authors propose that fosfomycin alters the membrane permeability of $P$. aeruginosa by affecting cell wall synthesis, which should lead to enhanced uptake of the fluoroquinolone ofloxacin (Monden et al., 2002). On the other hand, one study has suggested that the role of ciprofloxacin is thought to be related to damage of the outer membrane, enhancing fosfomycin penetration (Yamada et al., 2007). Regarding permeability in biofilms, it was reported that ciprofloxacin had a higher penetration rate $(>75 \%)$ than gentamicin $(73 \%)$ in $P$. aeruginosa biofilms (Abdi-Ali et al., 2006), but showed a similar kinetic of penetration than fosfomycin into the bacterial biofilm of both, E. coli and P. aeruginosa species (Rodríguez-Martínez et al., 2007). Based on these studies, it can be argued that all three antibiotics are able to penetrate well into biofilms. Thus, it could be hypothesized that the differences observed on the ability of each antibiotic combination to exert a synergistic effect might be attributed to other factors, such as killing of persister cells as proposed for streptococci in combinations including gentamicin (Gonzalez Moreno et al., 2017), rather than the enhancement of penetration. Further studies are required to clarify the underlying mechanism of their synergistic effect on biofilms.

The fosfomycin/gentamicin combination was the most active against E. coli strains. These results also correlate with the findings from Corvec et al., where fosfomycin plus gentamicin presented a significant high cure rate in an in vivo foreignbody infection model (Corvec et al., 2013). Moreover, the fosfomycin/gentamicin combination showed a synergistic effect toward a gentamicin-resistant strain, but the same combination was not synergistic toward a fosfomycin-resistant strain. Conventionally, the mechanism of action of gentamicin has been considered at the $30 \mathrm{~S}$ ribosomal level. Nevertheless, some authors have suggested that gentamicin has two potentially lethal effects on Gram-negative cells, one being the inhibition of protein synthesis and the other one being the surface perturbation (Kadurugamuwa et al., 1993a,b). Thus, a bacteriolysis effect mediated through perturbation of the cell surface by gentamicin could explain the synergism observed by the fosfomycin/gentamicin combination toward a gentamicinresistant strain. It could be speculated that, in the case of a gentamicin-resistant but fosfomycin-susceptible strain, while fosfomycin can act against susceptible bacterial cells, gentamicin could also actively target resistant bacterial cells through bacteriolysis, resulting in complete biofilm eradication. However, in the case of a gentamicin-susceptible but fosfomycin-resistant strain, no synergistic effect is observed because only gentamicin can act against bacteria, whereas fosfomycin becomes ineffective.
The two antimicrobial effects of gentamicin might also explain why combinations with this antibiotic seem to be particularly effective. Still, elucidations for synergistic effect based on planktonic findings would need to be confirmed also for biofilms.

In conclusion, the use of fosfomycin in combination with gentamicin seems to be a promising therapeutic approach against E. coli biofilm related infections. Nevertheless, against both Gramnegative species, combination of gentamicin with ciprofloxacin represents the most optimal treatment option. Further in vivo and clinical studies are essential to define the potential treatment regimen based on the combination of these two antibiotics. Moreover, our study presents IMC as a sensitive technique to provide reliable data on as important field of clinical microbiology as it is the screening for biofilm-eradicating approaches.

\section{DATA AVAILABILITY STATEMENT}

The datasets generated for this study are available on request to the corresponding author.

\section{ETHICS STATEMENT}

The clinical isolates were used from the biobank collection, which is part of the prospective institutional PJI cohort. The study was approved by the Institutional Ethical Committee (EA1/040/14) and was conducted in accordance with the most recent iteration of the Declaration of Helsinki. According to the ethical approval, participants' informed consent was waived and all data were pseudonymized.

\section{AUTHOR CONTRIBUTIONS}

LW, MG, MD, TT, and AT conceived and designed the experiments. LW performed the experiments. LW and MG analyzed the data. MG and LW drafted the manuscript, with the contribution of $\mathrm{MD}$ and AT. All authors reviewed and revised the final drafts of this manuscript.

\section{FUNDING}

This work was supported by the PRO-IMPLANT Foundation, Berlin, Germany (https://www.pro-implant-foundation.org). InfectoPharm supported this study by an educational grant.

\section{ACKNOWLEDGMENTS}

Part of the data was presented at the 29th European Conference of Clinical Microbiology and Infectious Diseases (Posters P0575 and P0572, Amsterdam, Netherlands, 13-16 April, 2019) and at the 38th European Bone and Joint Infection Society Conference (Oral presentation, Antwerp, Belgium, 12-14 September, 2019). We acknowledge support from the German Research Foundation (DFG) and the Open Access Publication Fund of Charité Universitätsmedizin Berlin. 


\section{REFERENCES}

Abdi-Ali, A., Mohammadi-Mehr, M., and Agha Alaei, Y. (2006). Bactericidal activity of various antibiotics against biofilm-producing Pseudomonas aeruginosa. Int. J. Antimicrob. Agents 27, 196-200. doi: 10.1016/j. ijantimicag.2005.10.007

Anderson, G. G., Kenney, T. F., Macleod, D. L., Henig, N. R., and O'toole, G. A. (2013). Eradication of Pseudomonas aeruginosa biofilms on cultured airway cells by a fosfomycin/tobramycin antibiotic combination. Pathog. Dis. 67, 39-45. doi: 10.1111/2049-632X.12015

Benito, N., Franco, M., Ribera, A., Soriano, A., Rodriguez-Pardo, D., Sorli, L., et al. (2016). Time trends in the aetiology of prosthetic joint infections: a multicentre cohort study. Clin. Microbiol. Infect. 22, e731-e738. doi: 10.1016/j.cmi.2016.05.004

Boyle, K. K., Kuo, F.-C., Horcajada, J. P., Hughes, H., Cavagnaro, L., Marculescu, C., et al. (2019). General assembly, treatment, antimicrobials: proceedings of international consensus on orthopedic infections. J. Arthroplast. 34, S225-S237. doi: 10.1016/j.arth.2018.09.074

Butini, M. E., Gonzalez Moreno, M., Czuban, M., Koliszak, A., Tkhilaishvili, T., Trampuz, A., et al. (2018). Real-time antimicrobial susceptibility assay of planktonic and biofilm bacteria by isothermal microcalorimetry. Adv. Exp. Med. Biol. 1-17. doi: 10.1007/5584_2018_291

Cai, Y., Fan, Y., Wang, R., An, M.-M., and Liang, B.-B. (2009). Synergistic effects of aminoglycosides and fosfomycin on Pseudomonas aeruginosa in vitro and biofilm infections in a rat model. J. Antimicrob. Chemother. 64, 563-566. doi: $10.1093 / \mathrm{jac} / \mathrm{dkp} 224$

CLSI (1999). "Methods for determining bactericidal activity of antimicrobial agents; approved guideline" in CLSI (eds). CLSI document M26-A (Wayne, PA: Clinical and Laboratory Standards Institute).

CLSI (2015). "Performance standards for antimicrobial susceptibility testing; twenty-fifth informational supplement" in CLSI (eds). CLSI document M100-S25 (Wayne, PA: Clinical and Laboratory Standards Institute).

Corvec, S., Furustrand Tafin, U., Betrisey, B., Borens, O., and Trampuz, A. (2013). Activities of fosfomycin, tigecycline, colistin, and gentamicin against extended-spectrum- $\beta$-lactamase-producing Escherichia coli in a foreign-body infection model. Antimicrob. Agents Chemother. 57, 1421-1427. doi: 10.1128/ AAC.01718-12

Dall, G. F., Tsang, S.-T. J., Gwynne, P. J., Mackenzie, S. P., Simpson, A. H. R. W., Breusch, S. J., et al. (2018). Unexpected synergistic and antagonistic antibiotic activity against Staphylococcus biofilms. J. Antimicrob. Chemother. 73, 1830-1840. doi: $10.1093 / \mathrm{jac} / \mathrm{dky} 087$

Dijkmans, A. C., Zacarías, N. V. O., Burggraaf, J., Mouton, J. W., Wilms, E. B., Van Nieuwkoop, C., et al. (2017). Fosfomycin: pharmacological, clinical and future perspectives. Antibiotics 6:24. doi: 10.3390/antibiotics6040024

Donlan, R. M., and Costerton, J. W. (2002). Biofilms: survival mechanisms of clinically relevant microorganisms. Clin. Microbiol. Rev. 15, 167-193. doi: 10.1128/CMR.15.2.167-193.2002

Falagas, M. E., Vouloumanou, E. K., Samonis, G., and Vardakas, K. Z. (2016). Fosfomycin. Clin. Microbiol. Rev. 29, 321-347. doi: 10.1128/CMR.00068-15

Fantoni, M., Borrè, S., Rostagno, R., Riccio, G., Carrega, G., Giovannenze, F., et al. (2019). Epidemiological and clinical features of prosthetic joint infections caused by Gram-negative bacteria. Eur. Rev. Med. Pharmacol. Sci. 23(Suppl. 2), 187-194. doi: 10.26355/eurrev_201904_17490

Goel, R., Tarabichi, M., Azboy, I., Kheir, M., and Parvizi, J. (2017). Management of periprosthetic joint infection. Rome, Italy: Edizioni Minerva Medica.

Gonzalez Moreno, M., Trampuz, A., and Di Luca, M. (2017). Synergistic antibiotic activity against planktonic and biofilm-embedded Streptococcus agalactiae, Streptococcus pyogenes and Streptococcus oralis. J. Antimicrob. Chemother. 72, 3085-3092. doi: 10.1093/jac/dkx265

Gonzalez Moreno, M., Wang, L., De Masi, M., Winkler, T., Trampuz, A., and Di Luca, M. (2019). In vitro antimicrobial activity against Abiotrophia defectiva and Granulicatella elegans biofilms. J. Antimicrob. Chemother. 74, 2261-2268. doi: $10.1093 / \mathrm{jac} / \mathrm{dkz} 174$

Greimel, F., Scheuerer, C., Gessner, A., Simon, M., Kalteis, T., Grifka, J., et al. (2017). Efficacy of antibiotic treatment of implant-associated Staphylococcus aureus infections with moxifloxacin, flucloxacillin, rifampin, and combination therapy: an animal study. Drug Des. Devel. Ther. 11, 1729-1736. doi: 10.2147/ DDDT.S138888
Haddad, F. S., Ngu, A., and Negus, J. J. (2017). Prosthetic joint infections and cost analysis? Adv. Exp. Med. Biol. 971, 93-100. doi: 10.1007/5584_2016_155

Izakovicova, P., Borens, O., and Trampuz, A. (2019). Periprosthetic joint infection: current concepts and outlook. EFORT Open Rev. 4, 482-494. doi: 10.1302/2058-5241.4.180092

Kadurugamuwa, J. L., Clarke, A. J., and Beveridge, T. J. (1993a). Surface action of gentamicin on Pseudomonas aeruginosa. J. Bacteriol. 175, 5798-5805.

Kadurugamuwa, J. L., Lam, J. S., and Beveridge, T. J. (1993b). Interaction of gentamicin with the A band and B band lipopolysaccharides of Pseudomonas aeruginosa and its possible lethal effect. Antimicrob. Agents Chemother. 37, 715-721.

Kastoris, A. C., Rafailidis, P. I., Vouloumanou, E. K., Gkegkes, I. D., and Falagas, M. E. (2010). Synergy of fosfomycin with other antibiotics for Grampositive and Gram-negative bacteria. Eur. J. Clin. Pharmacol. 66, 359-368. doi: 10.1007/s00228-010-0794-5

Kumar, C., Himabindu, M., and Jetty, A. (2008). Microbial biosynthesis and applications of gentamicin: a critical appraisal. Crit. Rev. Biotechnol. 28, 173-212. doi: 10.1080/07388550802262197

Kumon, H., Ono, N., Iida, M., and Nickel, J. C. (1995). Combination effect of fosfomycin and ofloxacin against Pseudomonas aeruginosa growing in a biofilm. Antimicrob. Agents Chemother. 39, 1038-1044. doi: 10.1128/AAC.39.5.1038

Li, C., Renz, N., and Trampuz, A. (2018). Management of periprosthetic joint infection. Hip. Pelvis 30, 138-146. doi: 10.5371/hp.2018.30.3.138

Macia, M. D., Rojo-Molinero, E., and Oliver, A. (2014). Antimicrobial susceptibility testing in biofilm-growing bacteria. Clin. Microbiol. Infect. 20, 981-990. doi: 10.1111/1469-0691.12651

Michalopoulos, A. S., Livaditis, I. G., and Gougoutas, V. (2011). The revival of fosfomycin. Int. J. Infect. Dis. 15, e732-e739. doi: 10.1016/j.ijid.2011.07.007

Mikuniya, T., Kato, Y., Ida, T., Maebashi, K., Monden, K., Kariyama, R., et al. (2007). Treatment of Pseudomonas aeruginosa biofilms with a combination of fluoroquinolones and fosfomycin in a rat urinary tract infection model. J. Infect. Chemother. 13, 285-290. doi: 10.1007/s10156-007-0534-7

Mikuniya, T., Kato, Y., Kariyama, R., Monden, K., Hikida, M., and Kumon, H. (2005). Synergistic effect of fosfomycin and fluoroquinolones against Pseudomonas aeruginosa growing in a biofilm. Acta Med. Okayama 59, 209-216. doi: 10.18926/AMO/31977

Monden, K., Ando, E., Kumon, H., and Iida, M. (2002). Role of fosfomycin in a synergistic combination with ofloxacin against $P$ seudomonas aeruginosa growing in a biofilm. J. Infect. Chemother. 8, 218-226. doi: 10.1007/s10156-002-0186-6

Rodríguez-Martínez, J. M., Ballesta, S., and Pascual, Á. (2007). Activity and penetration of fosfomycin, ciprofloxacin, amoxicillin/clavulanic acid and cotrimoxazole in Escherichia coli and Pseudomonas aeruginosa biofilms. Int J. Antimicrob. Agents 30, 366-368. doi: 10.1016/j.ijantimicag.2007.05.005

Rodriguez-Pardo, D., Pigrau, C., Lora-Tamayo, J., Soriano, A., Del Toro, M. D., Cobo, J., et al. (2014). Gram-negative prosthetic joint infection: outcome of a debridement, antibiotics and implant retention approach. A large multicentre study. Clin. Microbiol. Infect. 20, O911-O919. doi: 10.1111/ 1469-0691.12649

Sendi, P., Frei, R., Maurer, T. B., Trampuz, A., Zimmerli, W., and Graber, P. (2010). Escherichia coli variants in periprosthetic joint infection: diagnostic challenges with sessile bacteria and sonication. J. Clin. Microbiol. 48, 17201725. doi: 10.1128/JCM.01562-09

Shah, N. B., Osmon, D. R., Steckelberg, J. M., Sierra, R. J., Walker, R. C., Tande, A. J., et al. (2016). Pseudomonas prosthetic joint infections: a review of 102 episodes. J. Bone Jt. Infect. 1, 25-30. doi: 10.7150/jbji.15722

Sousa, R., and Abreu, M. A. (2018). Treatment of prosthetic joint infection with debridement, antibiotics and irrigation with implant retention - a narrative review. J. Bone Jt. Infect. 3, 108-117. doi: 10.7150/jbji.24285

Stewart, P. S. (2015). Antimicrobial tolerance in biofilms. Microbiol. Spectr. 3. doi: 10.1128/microbiolspec.MB-0010-2014

Taha, M., Abdelbary, H., Ross, F. P., and Carli, A. V. (2018). New innovations in the treatment of PJI and biofilms-clinical and preclinical topics. Curr. Rev. Musculoskelet. Med. 11, 380-388. doi: 10.1007/s12178-018-9500-5

Tangden, T., and Giske, C. G. (2015). Global dissemination of extensively drugresistant carbapenemase-producing Enterobacteriaceae: clinical perspectives on detection, treatment and infection control. J. Intern. Med. 277, 501-512. doi: 10.1111 /joim. 12342 
Thabit, A. K., Fatani, D. F., Bamakhrama, M. S., Barnawi, O. A., Basudan, L. O., and Alhejaili, S. F. (2019). Antibiotic penetration into bone and joints: an updated review. Int. J. Infect. Dis. 81, 128-136. doi: 10.1016/j.ijid.2019.02.005

Thai, T., and Zito, P. M. (2019). Ciprofloxacin. Treasure Island, FL, United States of America: StatPearls [Internet]: Treasure Island (FL): StatPearls Publishing.

Thompson, J. M., Miller, R. J., Ashbaugh, A. G., Dillen, C. A., Pickett, J. E., Wang, Y., et al. (2018). Mouse model of gram-negative prosthetic joint infection reveals therapeutic targets. JCI Insight 3:e121737. doi: 10.1172/jci. insight. 121737

Tucaliuc, D., Alexa, O., Tuchilus, C. G., Ursu, R. G., Tucaliuc, E. S., Jelihovsky, I., et al. (2015). Antibiotic resistance spectrum of non fermenting Gram negative bacilli isolated in the orthopedic traumatology clinic of "Sf. Spiridon" clinical emergency hospital Iasi. Rev. Med. Chir. Soc. Med. Nat. Iasi. 119, 536-543.

Virginio, C., Yuly, L., Estela, M., Dora, R., Carmen, A., Sara, M., et al. (2019). Relationship between biofilm formation and antimicrobial resistance in Gram-negative bacteria. Microb. Drug Resist. 25, 72-79. doi: 10.1089/ mdr.2018.0027

Walsh, C. C., Landersdorfer, C. B., Mcintosh, M. P., Peleg, A. Y., Hirsch, E. B., Kirkpatrick, C. M., et al. (2016). Clinically relevant concentrations of fosfomycin combined with polymyxin $\mathrm{B}$, tobramycin or ciprofloxacin enhance bacterial killing of Pseudomonas aeruginosa, but do not suppress the emergence of fosfomycin resistance. J. Antimicrob. Chemother. 71, 2218-2229. doi: 10.1093/ jac/dkw115

Wu, H., Moser, C., Wang, H. Z., Hoiby, N., and Song, Z. J. (2015). Strategies for combating bacterial biofilm infections. Int. J. Oral Sci. 7, 1-7. doi: 10.1038/ijos. 2014.65
Xiong, Y. Q., Potel, G., Caillon, J., Stephant, G., Jehl, F., Bugnon, D., et al. (1995). Comparative efficacies of ciprofloxacin and pefloxacin alone or in combination with fosfomycin in experimental endocarditis induced by multidrug-susceptible and -resistant Pseudomonas aeruginosa. Antimicrob. Agents Chemother. 39, 496-499. doi: 10.1128/AAC.39.2.496

Xu, X., Xu, L., Yuan, G., Wang, Y., Qu, Y., and Zhou, M. (2018). Synergistic combination of two antimicrobial agents closing each other's mutant selection windows to prevent antimicrobial resistance. Sci. Rep. 8:7237. doi: 10.1038/ s41598-018-25714-Z

Yamada, S., Hyo, Y., Ohmori, S., and Ohuchi, M. (2007). Role of ciprofloxacin in its synergistic effect with fosfomycin on drug-resistant strains of Pseudomonas aeruginosa. Chemotherapy 53, 202-209. doi: 10.1159/000100811

Zmistowski, B., Fedorka, C. J., Sheehan, E., Deirmengian, G., Austin, M. S., and Parvizi, J. (2011). Prosthetic joint infection caused by Gram-negative organisms. J. Arthroplast. 26, 104-108. doi: 10.1016/j.arth.2011.03.044

Conflict of Interest: The authors declare that the research was conducted in the absence of any commercial or financial relationships that could be construed as a potential conflict of interest.

Copyright (c) 2019 Wang, Di Luca, Tkhilaishvili, Trampuz and Gonzalez Moreno. This is an open-access article distributed under the terms of the Creative Commons Attribution License (CC BY). The use, distribution or reproduction in other forums is permitted, provided the original author(s) and the copyright owner(s) are credited and that the original publication in this journal is cited, in accordance with accepted academic practice. No use, distribution or reproduction is permitted which does not comply with these terms. 